\title{
Self-enhanced diffraction from fixed photorefractive gratings during coherent reconstruction
}

\author{
Mordechai Segev, Anthony Kewitsch, and Amnon Yariv \\ California Institute of Technology, The Thomas J. Watson, Sr., Laboratories of Applied Physics, \\ 128-95, Pasadena, California 91125 \\ George A. Rakuljic \\ Accuwave Corporation, 1653 19th Street, Santa Monica, California 90404
}

(Received 21 September 1992; accepted for publication 7 December 1992)

\begin{abstract}
We explore, theoretically and experimentally, the effects of self-enhancement (or self-depletion) of the diffraction which occurs during coherent reconstruction from fixed photorefractive gratings. These effects are caused by interference between a secondary grating, which forms between the readout and the reconstructed beams, and the fixed grating.
\end{abstract}

Photorefractive (PR) holograms can be fixed with various degrees of success. ${ }^{1-4}$ These usually involve the replacement of the original electronic charge by a relatively immobile ionic charge. When such holograms are reconstructed, by illuminating with the readout wave, a new dynamic electronic hologram is generated by the spatial interference of the readout and the diffracted waves. The superposition of the original (ionic) and the new (electronic) holograms, which are of the same period, can lead to self-enhancement ${ }^{1,2}$ or self-depletion, depending on their relative phase.

In this letter we revisit this problem theoretically and experimentally. Our analysis differs from previous ones, discussed by $\mathrm{Gu}$ and $\mathrm{Yeh}^{5,6}$ which examined a PR twowave mixing process in the presence of a fixed uniform grating. We consider a realistic case of a fixed PR grating, solve, rather than assume, for its spatially varying profile, and then derive the conditions for self-enhanced (and selfdepleted) diffraction. This enables us to draw quantitative conclusions and to compare our theoretical results to experiments using known material parameters. Finally, we demonstrate these effects experimentally for thermally fixed holograms in a PR $\mathrm{LiNbO}_{3}$ crystal, and show selfenhancement of the diffraction efficiency from $4 \%$ (of the fixed grating only) to $23 \%$.

We start the theoretical analysis by describing the holographic recording process, and assume an ordinary twowave mixing effect in a PR medium ${ }^{7}$ between two arbitrary input waves $a_{1}$ and $a_{2}$ (represented here by their field amplitudes) in the transmission geometry

$$
\begin{aligned}
& \frac{d a_{1}}{d z}=-\frac{\alpha}{2} a_{1}-i \Delta n a_{2}, \\
& \frac{d a_{2}}{d z}=-\frac{\alpha}{2} a_{2}-i \Delta n^{*} a_{1},
\end{aligned}
$$

where $\alpha$ is the intensity absorption coefficient, and $\Delta n$ the equivalent $P R$ perturbation in the refractive index,

$$
\Delta n=\gamma \frac{a_{1} a_{2}^{*}}{\left|a_{1}\right|^{2}+\left|a_{2}\right|^{2}} e^{-i \hat{\phi}}
$$

The factor $\gamma$ is the PR coupling coefficient, ${ }^{8}$ and $\hat{\phi}$ is the relative phase between the interference grating and the light-induced index grating (the so-called PR phase shift) during the recording process. Note that $\hat{\phi}$ may controlled by an externally applied electric field, and hence may differ from the PR phase shift during the reconstruction process. The boundary condition for Eqs. (1) and (2) are the input field amplitudes $a_{1}(0)$ and $a_{2}(0)$.

The fixing process involves a conversion of the electronic grating into an ionic one, hence the sign of the space-charge field (and therefore the signs of $\gamma$ and $\Delta n$ ) is reversed. The efficiency of the fixing process $\eta$ is usually less than unity. The resultant fixed grating is related to the recorded one by

$$
\Delta n_{\text {fixed }}=-\eta \Delta n=F(z) \exp \left[i\left(\phi_{0}-\hat{\phi}+\pi\right)\right],
$$

where

$$
F(z) \triangleq \eta \gamma \frac{\sqrt{\left|a_{1}\right|^{2}\left|a_{2}\right|^{2}}}{\left|a_{1}\right|^{2}+\left|a_{2}\right|^{2}}=\left|\Delta n_{\text {fixed }}\right|
$$

is a real and positive function of $z$, and $\phi_{0}=\hat{\phi}_{1}-\hat{\phi}_{2}$ is the relative phase between $a_{1}(z)$ and $a_{2}(z)$ in the recording process. The grating phase $\phi_{0}$ may vary with $z$ whenever the PR phase shift $\hat{\phi}$ is different than $\pm \pi / 2$.

We reconstruct this fixed PR grating with a Bragg matched readout wave $A_{1}$, parallel to $a_{1}$, and get a diffracted wave $A_{2}$ parallel to $a_{2}$ ( $A_{1}$ and $A_{2}$ are again the field amplitudes ). In a coherent reconstruction $A_{1}$ and $A_{2}$ interfere with each other and form an additional real-time PR grating. The coupled wave equations governing the combined scattering process, from the fixed and the real-time gratings, are

$$
\begin{aligned}
\frac{d A_{1}}{d z}= & -\frac{\alpha}{2} A_{1}-i F(z) \exp \left[i\left(\phi_{0}-\hat{\phi}+\pi\right)\right] A_{2} \\
& -i \gamma \frac{A_{1} I_{2}}{I_{0}} e^{-i \phi}, \\
\frac{d A_{2}}{d z}= & -\frac{\alpha}{2} A_{2}-i F(z) \exp \left[-i\left(\phi_{0}-\hat{\phi}+\pi\right)\right] A_{1} \\
& -i \gamma \frac{A_{2} I_{1}}{I_{0}} e^{i \phi},
\end{aligned}
$$

where $I_{i}(z)=\left|A_{i}(z)\right|^{2}, I_{0}=I_{1}+I_{2}$, where $A_{i}=\left|A_{i}\right| e^{-i \Phi_{i}}$ and $\phi$ is the PR phase shift in the readout process. The 
boundary conditions for the processes are either $\left[A_{1}(z=0)\right.$ $\left.=A_{10} ; A_{2}(z=0)=0\right]$, or the complementary set $\left[A_{1}(z=0)\right.$ $=0 ; A_{2}(z=0)=A_{20}$ ]. An equivalent set of equations, for the intensities and the phase of the waves, can be extracted from Eqs. (6) and (7):

$$
\begin{aligned}
\frac{d I_{1}}{d z}= & -\alpha I_{1}-2 F(z) \sqrt{I_{1} I_{2}} \sin \left(\phi_{0}-\hat{\phi}+\phi_{12}\right) \\
& -2 \gamma \frac{I_{1} I_{2}}{I_{0}} \sin \phi, \\
\frac{d I_{2}}{d z}= & -\alpha I_{2}+2 F(z) \sqrt{I_{1} I_{2}} \sin \left(\phi_{0}-\hat{\phi}+\phi_{12}\right) \\
& +2 \gamma \frac{I_{1} I_{2}}{I_{0}} \sin \phi, \\
\frac{d \phi_{1}}{d z}= & -\sqrt{\frac{I_{2}}{I_{1}}} F(z) \cos \left(\phi_{0}-\hat{\phi}+\phi_{12}\right)+\gamma \frac{I_{2}}{I_{0}} \cos \phi, \\
\frac{d \phi_{2}}{d z}= & -\sqrt{\frac{I_{1}}{I_{2}}} F(z) \cos \left(\phi_{0}-\hat{\phi}+\phi_{12}\right)+\gamma \frac{I_{1}}{I_{0}} \cos \phi,
\end{aligned}
$$

where $\phi_{i j}(z)=\phi_{i}(z)-\phi_{j}(z)$.

A special case is when the PR phase $\phi$ is equal to $\pm \pi / 2$ during both the recording and the reconstruction processes. Even though there is no phase coupling in the real-time PR process (in both the recording and the reconstruction), it still exists, owing to the diffraction off the fixed grating. Note that even in the case of diffraction from the fixed grating only, the coupling dynamics are asymmetric under the exchange of the readout beams.

The case of self-enhancement occurs when the coupling from both gratings adds up in phase. This happens according to Eqs. (6) and (7) when

$$
\phi_{0}(z)-(\hat{\phi}-\phi)+\phi_{12}(z)=(2 m+1) \pi,
$$

where $m$ is an integer, and self-depletion occurs for

$$
\phi_{0}(z)-(\hat{\phi}-\phi)+\phi_{12}(z)=2 m \pi \text {. }
$$

For the simple, special case of $\hat{\phi}=\phi=\pi / 2$ one gets the condition $\phi_{0}+\phi_{12}(z)=(2 m+1) \pi$ for self-enhancement (note that $\phi_{12}$ may vary with $z$ even in this case).

The fixed and the real-time gratings may differ not only in their relative phase, but also in their longitudinal distribution. While the real-time grating always starts with a zero modulation depth at $z=0$, the fixed grating may be $a$ priori designed and shaped according to the coupling process that takes place in the crystal, and the boundary conditions. In a case where the recording process is performed with waves of a polarization that corresponds to very low coupling, but the reconstruction is made with the polarization that yields a strong coupling coefficient, one can get a considerable self-enhancement with a "flat" fixed grating function $F(z)=$ constant. A similar experiment has been performed ${ }^{9}$ with a semipcrmanent fixed grating, recorded at an ordinary waves polarization in SBN, and reconstructed with an extraordinary polarization.

Extension of the above formulation, to include the time dynamics of the wave mixing process, can be straightfor-

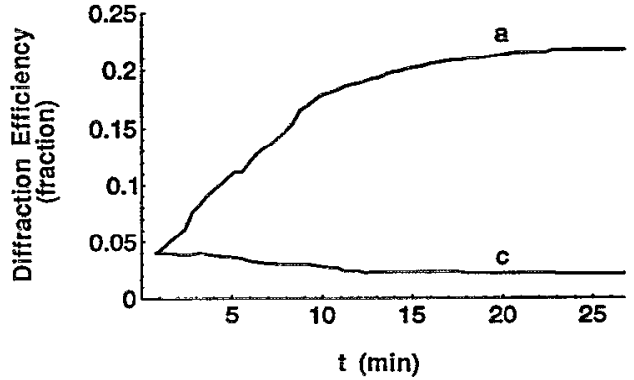

FIG. 1. Experimental results of the self-enhancement and self-depletion effects in $\mathrm{LiNbO}_{3}$.

wardly done following the guidelines of Ref. 10. However, since it is rather simple and involves only one time constant, we do not elaborate on the subject in this letter. We therefore compare only the steady-state theoretical and experimental results.

We studied experimentally the effects of selfenhancement in thermally fixed gratings in $\mathrm{LiNbO}_{3}$. The recording process was performed with input beams of identical intensities at $z=0$. The experimental results are presented in Fig. 1, where the temporal evolutions of the diffraction efficiencies of the diffracted beams are shown. The upper curve gives the time dependence of the diffraction efficiency for reconstruction with $A_{1}$, which yields a selfenhancement effect. The diffraction efficiency at $t=0$ is $4 \%$, identical to the diffraction efficiency of the fixed grating, and for large times it reaches a value of $23 \%$. The opposite dependence for the self-depleted diffraction efficiency, due to reconstruction with $A_{2}$, is presented by the lower curve of Fig. 1, where the same diffraction efficiency at $t=0$ was then depleted to a value of $0.8 \%$. After allowing the reconstruction to take place for a very long time ( $\sim 60 \mathrm{~min}$ ) both efficiencies were depleted due to Fanning effects ${ }^{11}$ and the reconstructed holograms were washed out completely by this stimulated noise. For a good discrimination between the reconstructed holograms and the fanning (which may generate amplified noise in the directions of the diffracted beams) we used image bearing beams for both the recording and the reconstruction holographic processes.

Our theoretical results were calculated with the boundary conditions $a_{1}(0)=a_{2}(0)$ in the recording process [Eqs. (1),(2)]. The material parameters were $\alpha=1.75 \mathrm{~cm}^{-1}$ and $\gamma=13 \mathrm{~cm}^{-1}$ and crystal length of $2.2 \mathrm{~mm}$. The best fit between the experimental and the theoretical model yielded $\eta=0.3$ and $\phi=\pi / 3$, and the results are presented in Fig. 2. Figure 2(a) shows the longitudinal $z$ evolution of the interacting beams in the reconstruction process. The upper solid curve in Fig. 2(a) ("b") shows the diffraction efficiency of the fixed grating (only) for reconstruction with $A_{1}$, and the upper dashed curve ("a") gives its selfenhanced diffraction efficiency. The diffraction efficiencies for reconstruction with $A_{2}$, of the fixed grating only, and the self-depletion are shown by the lower solid curve ("c") and the lowest dotted curve ("d"), respectively. Figure 2(b) shows the fixed grating function $F(z)$ (solid curve), 

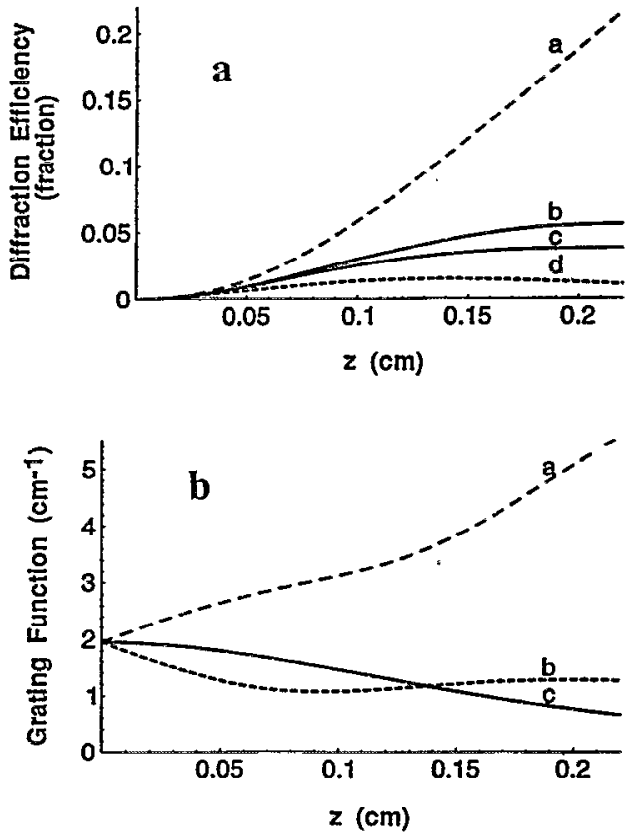

FIG. 2. Theoretical results of the longitudinal $z$ evolution of (a) the diffraction efficiencies and (b) the grating functions, for diffraction from the fixed (solid curves), self-enhanced (dashed curves), and self-depleted (dotted curves) gratings.

the real-time enhanced grating (dashed curve), and the real-time depleted grating (dotted curve).

Finally, we suggest that the material's diffraction efficiency may be increased to a value that exceeds the one of a recording process (or, equivalently, to increase the effective $\gamma$ ). Using the fact that the fixed and the real-time gratings in a coherent reconstruction process utilize differ- ent sources of space charges, their peak values are not limited by the maximum trap density, as in real-time gratings. In the optimal case, where the grating function is a constant and the fixing efficiency is unity, one expects the density of ions that move (and compensate for the electronic field) to be identical to the trap density. The selfenhancement process redistributes the electrons in the vacant traps in such a way that both gratings are in phase with each other, and the diffraction efficiency reaches a higher value. Since the current values for diffraction efficiency from fixed gratings are relatively high, ${ }^{4}$ one may use this grating enhancement to shorten the crystal length while keeping the diffraction efficiency close to unity. Efficient holographic storage at a shorter crystal length facilitates larger numerical apertures together with smaller absorption losses, which is desirable for many applications.

This work was supported by the Defense Advanced Projects Agency (DARPA), the Army Research OfficeDurham, and by Air Force Office of Scientific Research. A. $K$. acknowledges the support of an ONR (Office of Naval Research) Fellowship.

${ }^{1}$ J. J. Amodei and D. L. Staebler, Appl. Phys. Lett. 18, 540 (1971).

${ }^{2}$ D. L. Staebler and J. J. Amodei, Ferroelectrics 3, 107 (1972).

${ }^{3}$ V. Leyva, A. Agranat, and A. Yariv, Opt. Lett. 16, 554 (1991), and references therein.

${ }^{4}$ G. A. Rakuljic and A. Yariv (unpublished).

${ }^{5}$ C. Gu and P. Yeh, J. Opt. Soc. Am. B 7, 2339 (1990).

${ }^{6}$ C. Gu, J. Hong, and P. Yeh, J. Opt. Soc. Am. B 9, 1473 (1992).

${ }^{7}$ D. L. Staebler and J. J. Amodei, J. Appl. Phys. 43, 1042 (1972).

${ }^{8}$ A. Yariv, Optical Electronics, 4th ed. (Saunders College Publishing, 1991), Chap. 18.

${ }^{9}$ S. Redfield and L. Hesselink, Opt. Lett. 13, 880 (1988).

${ }^{10}$ G. C. Valley, J. Opt. Soc. Am. B 9, 1440 (1992).

${ }^{11}$ M. Segev, Y. Ophir, and B. Fischer, Opt. Commun. 77, 265 (1990). 